\title{
SILICON NANOWIRES AS BIOCOMPATIBILE ELELCTRONICS-BIOLOGY INTERFACE
}

\author{
Paola Piedimonte ${ }^{I^{*}}$, Sergio Fucile ${ }^{2,3}$, Cristina Limatola ${ }^{2,3}$, Massimiliano Renzi $^{2}$ and Fabrizio Palma ${ }^{1}$ \\ ${ }^{1}$ Basic and Applied Sciences for Engineering Dep., Sapienza University of Rome, 00184 Rome, Italy \\ ${ }^{2}$ Physiology and Pharmacology Dep., Sapienza University of Rome, 00184 Rome, Italy and \\ ${ }^{3}$ IRCCS Neuromed, Pozzilli, Italy
}

\begin{abstract}
Silicon nanowires (SiNWs) represent new opportunities for developing electrical biosensors due to their inherent properties, including large surface-tovolume ratio, rapid signal response and nanoscale footprint comparable to biomolecular and subcellular structures. Still, fabrication of nanosized electrodes is time-consuming, pricey and might be only scarcely compatible with the Complementary-Metal-OxideSemiconductor integrated circuits (CMOS-IC) technology. To take a step forward, we introduced an innovative approach to fabricate small, high-density SiNWs with a low-temperature $\left(200{ }^{\circ} \mathrm{C}\right)$ and CMOScompatible method. In this work, the fabrication process and the preliminary results showing biocompatibility and neutrality of SiNWs used as seeding substrate for cultured cells are presented.
\end{abstract}

\section{KEYWORDS}

Silicon nanowires, CVD, biointerface, biosensor, CMOS compatible, Vapor-Liquid-Solid growth.

\section{INTRODUCTION}

Recently, there has been an increasing progress in developing nanotechnologies for biological applications. These technologies can be defined as functional devices or structures with at least one dimension on the nanoscale $(\sim 100 \mathrm{~nm})$. Critical components involved in their production can include bottom-up synthesis of nanostructures, manipulation and/or assembly of these structures, and nano-fabrication methods, which can define and connect structures on a nanoscale and larger (i.e. chips). New developments are reaching a level of sophistication that may impact standard electrophysiological recording methods, such as patch clamp. In fact, in most configurations patch-clamp relies on accessing (thus perturbing) the interior of single cells, which limits the recording output both in duration and overall number of examined samples. In order to overcome such limitations, different extracellular recording methods (e.g. Multi Electrode Arrays (MEA) [1] and multi-transistor arrays [2]) have been explored. However, these methods sacrifice the direct correspondence between cells and electrodes and might offer recordings with low signal-to-noise ratio. A valid alternative is represented by optical methods based on ion indicators and voltage-sensitive dyes [reviewed in 3]. With these technologies it's possible to perform parallel recordings, yet they might still suffer for perfectible resolution and applicability. Overall, both pharmacological screening of ion-channel drugs and highresolution investigation of cell excitability are still usually executed by means of low-throughput, intracellular recording methods [reviewed in 4].

In this scenario, assembling an all-electrical device for electrophysiological imaging has long been a major pursuit in bioengineering applied to neuro- and cardioexperimental physiology [5]. Notably, one of the most important challenge for this approach is represented by the interface between the cell membrane and the nanoelectrodes which is affected by the electrical screening of biological signals along even very short distance (e.g. $\sim 10$ nm) [6]. Recently, adoption of nanowire transistors [7], nanotube-coupled transistors [8] and laser-induced optoporation MEA [9] did pave the way to a new technology with significantly improved signal resolution during recordings from various living cells. Nano-electrodes with different geometries based on CMOS-ICs could be used to record action potentials from cardiomyocytes in culture over a long period of time with an upgraded signal resolution [10]. Still, CMOS-based technology and nanoscaled electrodes have not been combined yet into an all electrical, low-cost device due to the scarce compatibility between CMOS technology and the nanotechnology required to grow on site nanowires. Trying to fill this gap, we designed a new approach to fabricate nano-scaled, high-density and CMOS-compatible silicon nanowires. The possibility to growth such nano-detectors directly onto the ICs allows an extremely tight coupling between the cell membrane and the recording device, theoretically minimizing the electrical screening of biological signals.

By introducing this new technology, we hope to overcome the limitations suffered insofar in the field and aim to produce a device for massively parallel electrophysiological imaging of biological samples at high-resolution.

\section{FABRICATION}

\section{Silicon Nanowires Growth}

So far, the two major techniques developed for nanowires preparation have been the "top-down" and "bottom-up." For top-down technique, nanowires are shaped from the bulk of Si wafers and patterned through microfabrication processes (e.g. lithographic techniques combined with dry and wet etching) [11]. The production of bottom-up nanowires starts with the growth of SiNWs, which are normally grown on the substrate with the help of metal catalysts. Our SiNWs were synthesized with a bottom-up technique using the Vapor Liquid Solid (VLS) mechanism (first demonstration of this mechanism in 1964 [12]). The main advantage of using this technique is the possibility to customize the process parameters in order to obtain the desired nanowires features. In this case, nanowires diameter can be tailored by the sizes of 
the metal-catalyst clusters. Indeed, for our technique the metal droplets formed do function as nano-susceptors for nanowires which in turn, once the metal-semiconductor eutectic temperature has been overcome, build-up in presence of inflowing silane. So, the metal catalyst choice is crucial. The most commonly used metal catalyst for silicon wires growth has been gold and it is still frequently employed [13,14]. However, many efforts have been made to find a valid alternative, as using Au induces deep level defects in the Si structure [15], thus making this catalyst scarcely compatible with the CMOS technology standards. For our deposition process, Sn has been chosen as catalysts material. According to [16], both the impurity levels in $\mathrm{Si}$ bandgap and minimum temperature for VLS growth meet the compatibility requirements.

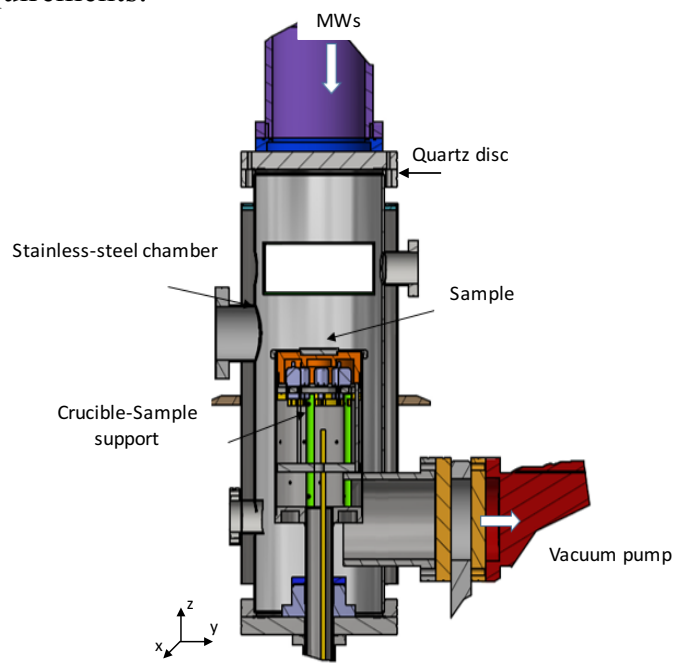

Figure 1: Schematic of the MWCVD deposition chamber. The substrate with the Sn layer is located on the crucible support and due to the tangential electric field focused on its surface, Sn droplets are heated up by MWs and work as nano-susceptors.

Figure 1 shows the deposition chamber. The main structure is a cylindrical tube used as waveguide for TE10 mode propagation of the electromagnetic field generated by a magnetron at $2.5 \mathrm{GHz}$. Since the growth is performed in high vacuum conditions, a quartz disc is interposed between the MW generator and the chamber, allowing MW passage while guaranteeing the preservation of deposition chamber settings. The sample holder is positioned at the bottom of the whole structure and can be controlled along $\mathrm{z}$-axis in order to modify the waveguide conditions. In order to locally damp the intensity of the generated field and to prevent energy dissipation towards the conducting crucible, a nitride spacer is positioned between the crucible surface and the sample substrate.

First step for SiNWs fabrication was the substrate baking in vacuum conditions $\left(1 \times 10^{-6} \mathrm{mbar}\right)$ and at $400{ }^{\circ} \mathrm{C}$ with a $3200{ }^{\circ} \mathrm{C} / \mathrm{h}$ regime. Those conditions, thanks to the coalescence mechanism, ensure the establishment of small Sn droplets $(\sim 30 \mathrm{~nm})$ on the substrate surface as reported in Figure 2 (a). For all the heating steps, the temperature was monitored using a thermocouple in contact with the backside of the sample holder. After the baking, the substrate was cooled to $200^{\circ} \mathrm{C}$ and exposed to a $\mathrm{SiH}_{4}$ environment for several minutes in order to obtain the desired nanowires shape and length. The resulting nanowires are depicted in Figure 2 (b).

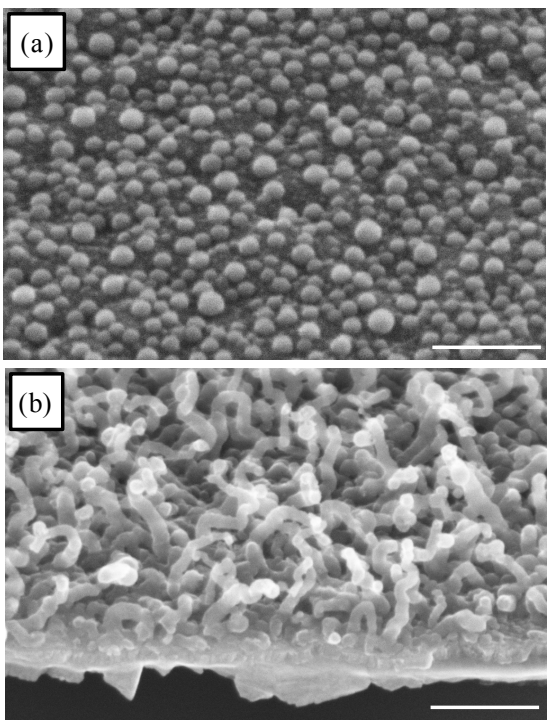

Figure 2: SEM images of two deposition steps. In (a), Sn droplets on Si substrate before the deposition process ( $\sim 30 \mathrm{~nm}$ diameter) and in (b) SiNWs after the full fabrication procedure (20-30 $\mathrm{nm}$ diameter). In (b) the metal droplet is still evident at nanowires tips. (Scale bars: $(a, b) 100 \mathrm{~nm})$.

\section{Cell Cultures, Patch-clamp, $\mathrm{Ca}^{2+}$ imaging and SEM}

Cell lines were grown in standard conditions $(10 \% \mathrm{FBS}$, $1 \% \mathrm{P} / \mathrm{S} \mathrm{DMEM}$ ), plated on uncoated substrates (glass coverslips or SiNWs) and used after 24-48 hrs. Primary cultures from neonatal mice were kept in standard conditions for 11 days (neurons) or 7 days (mixed glial culture). Microglia was seeded on SiNWs after purification from the mixed glial culture. In patch-clamp experiments we used standard external (NaCl-based) and pipette (K-gluconate-based) solutions to investigate passive and active neuronal properties and the expression of voltage-activated Inwardly or Outwardly Rectifying (IR or OR) $\mathrm{K}^{+}$channels in microglial cells. To quantify the peak-current density, the cell capacitance was estimated from the amplifier capacitance compensation dial. For $\mathrm{Ca}^{2+}$ imaging, we loaded $\mathrm{BV}-2$ cells with the $\mathrm{Ca}^{2+}$-sensitive fluorescent dye Fura- $2(2 \mu \mathrm{M})$ and recorded the epifluorescence $(380 \mathrm{~nm}$ exc., $510 \mathrm{~nm}$ em. wavelengths) time course in response to the fast application of $1 \mathrm{mM}$ ATP (3-sec long application). Cells grown on nanowires were prepared for SEM using a standard protocol consisting of fixation in glutaraldehyde (2,5\% in PBS for $40 \mathrm{~min}$ at room temperature) and sample dehydration in rising concentrations of ethanol followed by overnight treatment in Hexamethyldisilazane. Fixed substrates were coated with few nanometers of chromium (sputtering) to increase SEM contrast. All recordings were at room temperature. Results are shown as mean \pm s.e.m. 


\section{RESULTS}

The cellular membrane/nanostructures interface regulates all the biological processes and it's crucial for design of bio-devices. Despite the fact that recent advancements in the fabrication of artificial bio-interfaces have yielded an enhanced understanding of this interface, there remain open questions on how the cellular membrane behaves in the presence of sharp objects at the nanoscale. Therefore, to first characterize this behavior in a fixed condition, a SEM characterization of cell membrane-nanowires interaction has been made.

In Figure $3 \mathrm{SEM}$ images of $\mathrm{BV}-2$ cell cultures on SiNWs are showed. As a first observation, we notice that the SEM image resolution and contrast are sufficient to discern clearly between the cell/cell membrane and the SiNWs. More importantly, the overall cell morphology appears unaltered and the cellular membrane can be seen adhering to the substrates very tightly (Figure $3, \mathrm{~b}$ ). The images show that independent of the nanostructure size and orientation the cellular membrane tends to adhere to the substrate along the full profile, including the sharp edge at nanowires base.
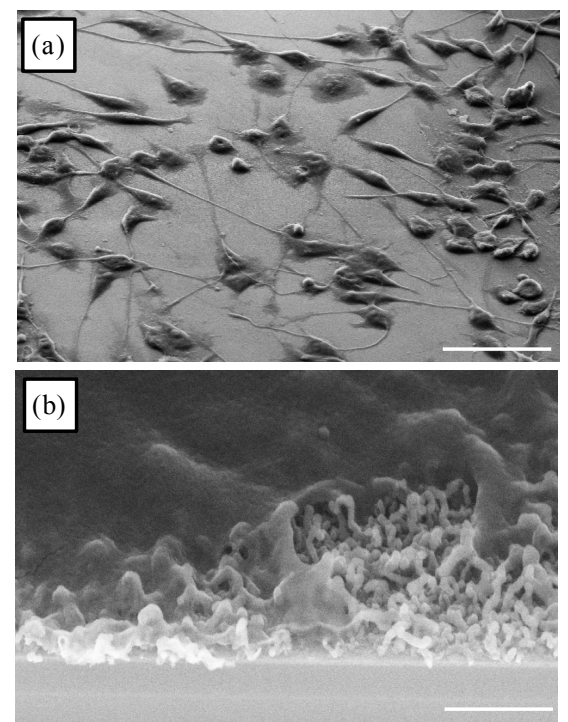

Figure 3: SEM images of BV-2 cell on SiNWs. (a) shows a portion of cell culture (scale bar $20 \mu \mathrm{m}$ ). The magnification (b) demonstrates the intimate contact between the cell and the nano-structures (scale bar: 200 nm).

As BV-2 cells were successfully grown on engineered substrates we stepped forward and tested their physiological properties using patch-clamp and $\mathrm{Ca}^{2+}$ imaging. Thus, to investigate whether SiNWs could alter the pattern of $\mathrm{K}^{+}$ion channels expressed on these cells (compared to glass coverslips as standard substrates; [17]) we recorded whole-cell membrane currents elicited by voltage steps and quantified their peak-current density. No apparent difference was found between control (Ctrl) and silicon substrates ( 3 and 4 cells, respectively; Figure 4 , a). To further demonstrate that cells grown on SiNWs do express a pattern of membrane receptors similar to those present in physiological conditions we performed $\mathrm{Ca}^{2+}$ imaging experiments on BV-2 cells on SiNWs and found that both basal intracellular $\left[\mathrm{Ca}^{2+}\right]$ and $1 \mathrm{mM}$ ATPelicited $\left[\mathrm{Ca}^{2+}\right]_{\mathrm{i}}$ rise were typical of these cells in normal culture conditions (Figure 4, b; [18]). In line with these findings, primary microglia from neonatal mice could be grown on SiNWs with no apparent alteration of their morphology (Figure 4, c).

(a) Patch-clamp
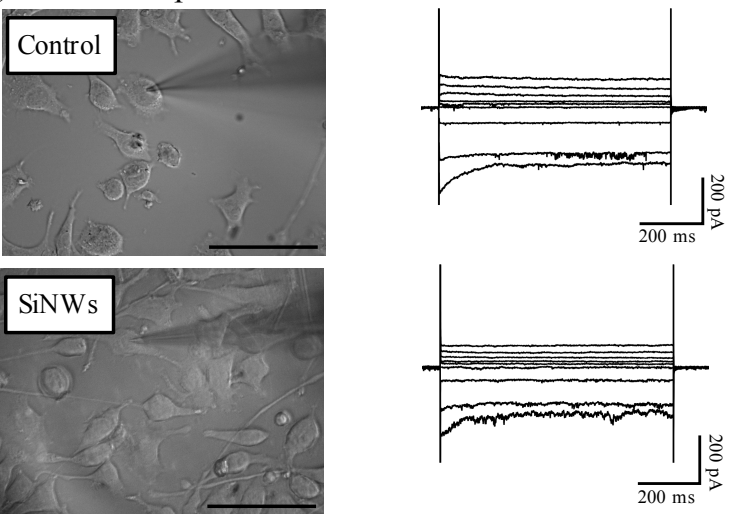

(b) Calcium imaging
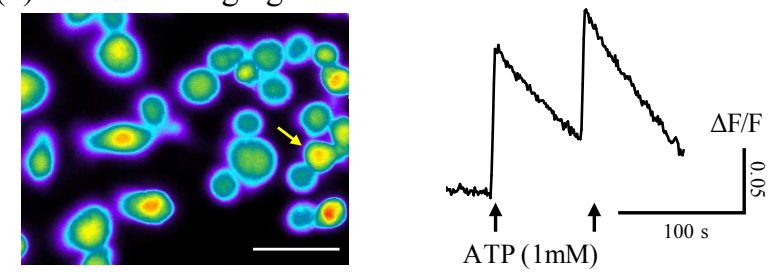

(c) Immunofluorescence
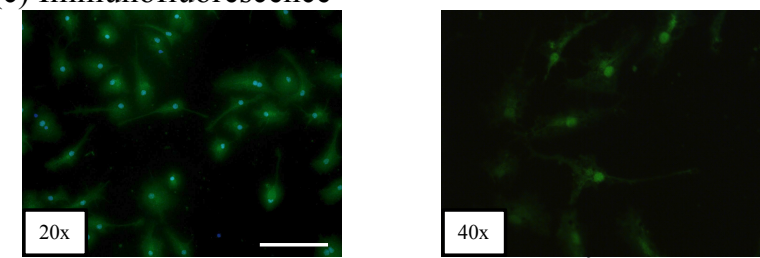

Figure 4: Voltage-clamp recordings, $\mathrm{Ca}^{2+}$ imaging and immunofluorescence on microglial cells. (a) typical transmitted (IR-DIC) image of BV-2 cells on SiNWs (left) and current response to applied voltage steps $\left(V_{c m d}-130\right.$ to $+30 \mathrm{mV}$, 1-sec long, $20 \mathrm{mV} / \mathrm{step}$; holding potential -70 $\mathrm{mV}$. (c) typical optic field depicting fura-2 AM loaded cells (left). The arrow indicates a cell responsive to the fast application of $1 \mathrm{mM}$ ATP. Right, time course of the fluorescence response (indicating $\left[\mathrm{Ca}^{2+}{ }_{i}\right]$ rise) to two consecutive applications of ATP (arrows). Typically, we found three-to-four responsive cells per optical field (6 fields analyzed across different substrates, no difference found). (c) Magnifications of immunofluorescence depicting microglial cells in culture on SiNWs (green, anti-phalloydin labelling microglia; blue, Hoechst labeling every cell nuclei). (All scale bars: $25 \mu \mathrm{m}$ )

Similar to microglial cells, also neuronal cells could grow on SinWs. We cultured NG108CC15 cells, a hybrid cell line showing neuronal properties [19] and in currentclamp experiments we found that in response to steps of hyperpolarizing or depolarizing injected currents NG cells had both passive properties (resting membrane potential; membrane capacitance; voltage sag and input resistance) and active response (firing profile) unaltered by the presence of SiNWs (3-to-4 cells; Figure 5, a). SiNWs were also tested for biocompatibility using primary hippocampal neurons from neonatal mice with no 
apparent alteration of their morphology (Figure 5, b).

(a) Patch-clamp
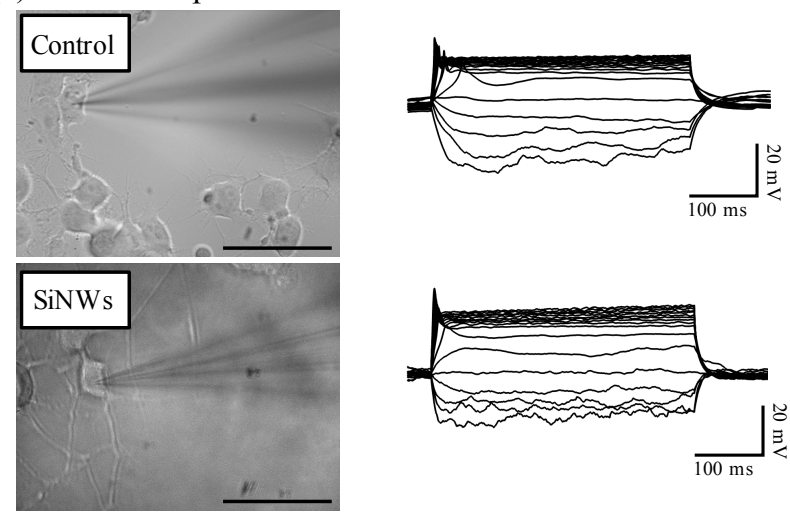

(b) Immunofluorescence
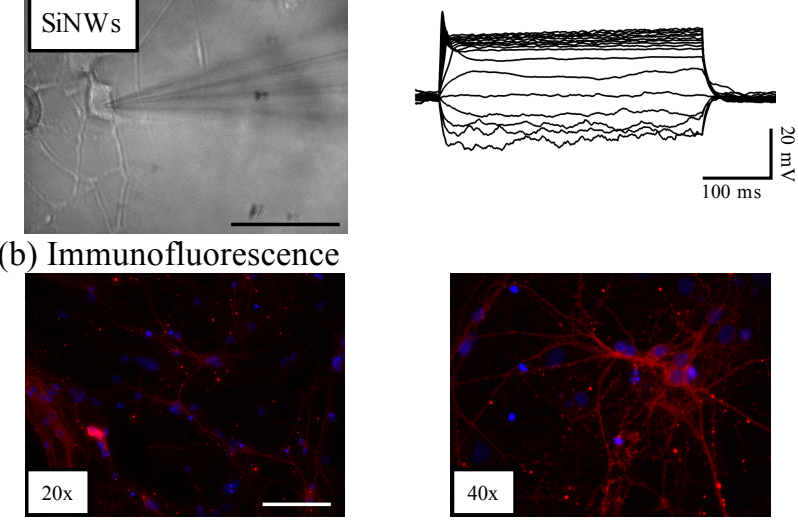

Figure

5:

Current-clamp

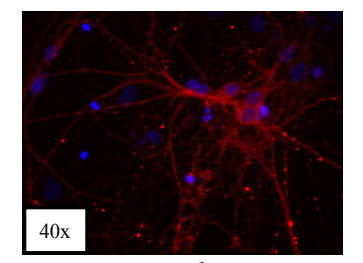

recordings and immunofluorescence of neuronal cells. (a) Typical IR-DIC images of NG18CC15 cells (left) and their voltage response to steps of injected current (right) showing unaltered morphology and electrophysiological properties in presence of different seeding substrates. Injected current steps: $I_{i n j}-200$ to $+600 \mathrm{pA}, 50 \mathrm{pA}$ increments, $1 \mathrm{~s}$-long; inter-sweep-interval $1.5 \mathrm{sec}$; $\mathrm{HP}-80$ $m V$. (b) Mouse hippocampal neurons after 11 days in culture on uncoated SiNWs (red, anti-TuJl labeling neurons; blue, Hoechst labeling every cell nuclei). (All scale bars: $25 \mu \mathrm{m}$ )

\section{CONCLUSIONS}

We described a novel technology for the fabrication of small, high-density Silicon NanoWires, which can theoretically be grown directly on CMOS IC-equipped substrates. Our method is fast, inexpensive and works at relatively low temperature. Of note, using a multidisciplinary approach (SEM; patch-clamp; $\mathrm{Ca}^{2+}$ imaging) we demonstrated that our SiNWs are neutral to living cells and thus potentially amenable to pass electric signals both from and onto cells in tight contact.

\section{ACKNOWLEDGEMENTS}

The authors acknowledge the Center for Nanotechnology Applied to Engineering (CNIS) of Sapienza University of Rome for the FE-SEM and Dr G. D'Alessandro for her help with immunofluorescence on primary cultures.

\section{REFERENCES}

[1] J. Pine, "Recording action potentials from cultured neurons with extracellular microcircuit electrodes.", J. Neurosci. Methods 2(1), 19 (1980). [2] A. Lambacher et al., "Electrical imaging of neuronal activity by multi-transistor-array recording at 7.8 $\mu \mathrm{m}$ resolution.", Applied Physics A 79, 1607-1611
(2004).

[3] D. S. Peterka et al., "Imaging voltage in neurons.", Neuron 69(1), 9 (2011).

[4] A. Obergrussberger et al., "Novel screening techniques for ion channel targeting drugs.", Channels (Austin) 9(6), 367 (2015).

[5] P. Fromherz, "Electrical interfacing of nerve cells and semiconductor chips.", Chem. Phys. Chem. 3(3), 276 (2002).

[6] E.R. Fossum et al., "A review of the pinned photodiode for CCD and CMOS image sensors.", IEEE J.

Electron Devices Soc. 2(3), pp 33-43 (2014).

[7] B. P. Timko et al., "Electrical recording from hearts with flexible nanowire device arrays.", Nano Letters, 9(2), 914 (2009).

[8] X. Duan et al., "Intracellular recordings of action potentials by an extracellular nanoscale field-effect transistor.", Nature Nanotechnology 7(3), 174 (2011).

[9] M. Dipalo et al., "Intracellular and Extracellular Recording of Spontaneous Action Potentials in Mammalian Neurons and Cardiac Cells with 3D Plasmonic Nanoelectrodes", Nano Letters 17(6), 3932 (2017).

[10] J. Abbott et al., "CMOS nanoelectrode array for all-electrical intracellular electrophysiological imaging.", Nature Nanotechnology 12(5), 460 (2017).

[11] I. Park et al., "Top-down fabricated silicon nanowire sensors for real-time chemical detection.", Nanotechnology 2, 015501 (2010).

[12] R. S. Wagner et al., "Vapor-Liquid-Solid Mechanism of Single Crystal Growth.", Applied Physics Letters 4(5), 89 (1964).

[13] W. Chen et al., "Atomic characterization of $\mathrm{Au}$ clusters in vapor-liquid-solid grown silicon nanowires.", Journal of Applied Physics 118(10), 104301 (2015).

[14] A. Convertino et al., "Disordered array of Au covered Silicon nanowires for SERS biosensing combined with electrochemical detection.", Scientific Reports 6, 25099 (2016).

[15] J. E. Allen et al., "High-resolution detection of Au catalyst atoms in Si nanowires.", Nature Nanotechnology 3, 168 (2008).

[16] B.V. Schmidt et al., "Silicon Nanowires: A Review on Aspects of their Growth and their Electrical Properties", Advanced Materials 21, 2681-2702 (2009). [17] S. Visentin et al., "Altered outward-rectifying $\mathrm{K}^{+}$ current reveals microglial activation induced by HIV-1 Tat protein.”, Glia 33(3), 181 (2001).

[18] L. P. Bernier et al., "Phosphoinositides Regulate P2X4 ATP-Gated Channels through Direct Interactions", Journal of Neuroscience 28(48), 12938 (2008).

[19] J. Liu et al., "Voltage-gated sodium channel expression and action potential generation in differentiated NG108-15 cells", BMC Neuroscience 13(1), 129 (2012).

\section{CONTACTS}

*P. Piedimonte, tel: +39 0644585357

paola.piedimonte@uniroma1.it

DIET, Via Eudossiana 18, 00184 Rome, Italy 